\title{
Pathological findings of central nervous system, two GM1 gangliosidosis autopsy cases
}

\author{
Doğuş Özdemir Kara ${ }^{1}$, Ahmet Şahpaz ${ }^{2}$ \\ ${ }^{1}$ Department of Pathology, Ankara Branch of the Council of Forensic Medicine, Ankara; ${ }^{2}$ Department of Pathology, Erzurum \\ Branch of the Council of Forensic Medicine, Erzurum, Turkey.E-mail: dogusdr@yahoo.com \\ Received: 31st October 2018, Revised: 27th December 2018, Accepted: 23rd January 2019
}

SUMMARY: Özdemir Kara D, Şahpaz A. Pathological findings of central nervous system, two GM1 gangliosidosis autopsy cases. Turk J Pediatr 2019; 61: 953-957.

GM1 gangliosidosis is an autosomal recessive lysosomal storage disease which is characterized by the accumulation of GM1 ganglioside, sphingolipids, glycoprotein bound oligosaccharides and keratan sulphate. Three major clinical forms have been identified depending on the enzyme levels, the timing of onset and severity of the manifestations; infantile form being the most severe. We herein present the clinical features and the histopathological findings of the central nervous system of two cases with type I infantile gangliosidosis; the first one had been diagnosed when she was 8 months old and died at the age of 3; the second one had been diagnosed when he was 10 months old and died at the age of 2 . Correlations between clinical features and histopathological findings have been discussed. Postmortem examination of both cases revealed a severe cortical thinning of cerebrum and cerebellum, accompanied by ventricular dilatation. Light microscopic evaluation of cerebral, cerebellar and brainstem sections demonstrated a distortion of normal parenchymal structures and presence of cellular aggregates bearing large, foamy and eosinophilic cytoplasm. Clinical records of the cases revealed a history of delayed mental and motor development, and frequently recurring infection episodes for both cases. Postmortem histopathological examination of such cases enables us to thoroughly identify and describe the multisystemic organ pathologies stemming from gangliosidosis. In this report, we aim to put an emphasis on the specific postmortem neuropathological findings in GM1 gangliosidosis. For a definitive diagnosis, documentation of low ß-galactosidase enzyme levels in leucocytes is a must.

Key words: GM1 gangliosidosis, autopsy, pathologic findings.

GM1 gangliosidosis is an autosomal recessive lysosomal storage disease which is characterized by the accumulation of GM1 ganglioside, sphingolipids, glycoprotein bound oligosaccharides and keratan sulphate. The enzyme-encoding gene is located in the 3 p21 chromosomal region. Although its true incidence is not known, it is estimated to be about 1 in 100,000-200,000 newborns. ${ }^{1}$ Three major clinic forms have been identified depending on the enzyme levels, the timing of clinical onset and severity of the symptoms: type 1, the infantile form; type 2 , the lateonset infantile-juvenile form; and type 3 , the adult form. ${ }^{2}$ Infantile form is the most severe of all with an early clinical onset.

Early symptoms and findings of type 1 gangliosidosis may become apparent during the neonatal period, although they typically manifest between 6-18 months of age. The most commonly encountered presentations that are suggestive of GM1 gangliosidosis include psychomotor retardation, hypotonia, hepatosplenomegaly, edema, coarse facial features, skeletal deformities, and macular cherry-red spot. ${ }^{3}$ Infantile cases generally suffer convulsions, rapid neurological deterioration, 
and eventually early childhood deaths. ${ }^{4}$ Herein we report two cases of infantile type I gangliosidosis with early childhood onset of motor/mental developmental abnormalities and with rapid neurologic deterioration. The primary cause of morbidity and mortality was the frequently recurring infection episodes as a consequence of nutritional problems and impaired respiratory functions.

Post-mortem gross central nervous system examination results and histopathological findings were significant and well correlated with the rapidly progressive clinical course. Histopathological marks of the disease were also noted in other organs and systems.

\section{Case Reports}

\section{Case 1}

Our first case was a girl, who had been diagnosed as type I GM1 gangliosidosis when she was 8 months old upon her admission to a hospital for developmental delay. During her follow-up period for about two years, according to her previous clinical records, she had undergone frequent hospitalizations for recurrent lung infections. Hypertrophic cardiomyopathy and pulmonary hypertension were also present, as detected by echocardiography. She died at the age of 3 due to cardiopulmonary arrest.

Her clinical and family history revealed that her mother was 18 years old when the patient was born full-term after a normal pregnancy period without any gestational problems. One of her first cousins had also suffered and died of the same disorder.

The autopsy was performed in our institution. On external physical examination, a facial dysmorphism was remarkable with a flat and large nasal bridge, frontal bossing, sunken eyes, macrocephaly, and occipital flattening possibly due to prolonged supine position. As for the internal evaluation; cerebrum and cerebellum weighed $1,575 \mathrm{~g}$, serial sections of which showed prominent cortical thinning with a thickness of $0.3 \mathrm{~cm}$, and areas of intensive hyperemia and pinpoint hemorrhage within substantia alba. The ventricles were extensively dilated, and corpus callosum was detected to be in a membranous morphology with a thickness of $0.2 \mathrm{~cm}$ at its maximal width. Intraabdominal examination demonstrated a firm and enlarged liver weighing 1,000 g. Serial sections of the liver showed several yellow spotty areas, being more pronounced around the portal vein. Neither a gross pathology of other organs nor an evidence of intoxication was detected.

Histopathological evaluation of the hematoxylin and eosin (H\&E) stained brain tissue sections revealed that normal brain parenchyma was replaced by a group of large cells having a foamy and eosinophilic cytoplasm. Cerebellar tissue sections demonstrated a distinct distortion of the usual molecular and granular cell layers, as well as a diminished number and intensity of Purkinje cells (Fig. 1). The aforementioned large foamy and eosinophilic cell groups were also detected in the brain stem sections (Fig. 2). Evaluation of the hepatic sections showed that the normal parenchymal construction had been completely lost and divided by fibrotic bands. A pronounced micro- and macrovesicular steatosis were present, and cellular groups with large and foamy cytoplasm were detected within the fibrotic areas.

\section{Case 2}

The second case was a boy, who had been diagnosed as type I infantile GM1 gangliosidosis when he was 10 months old and had died of cardiopulmonary arrest at the age of two.

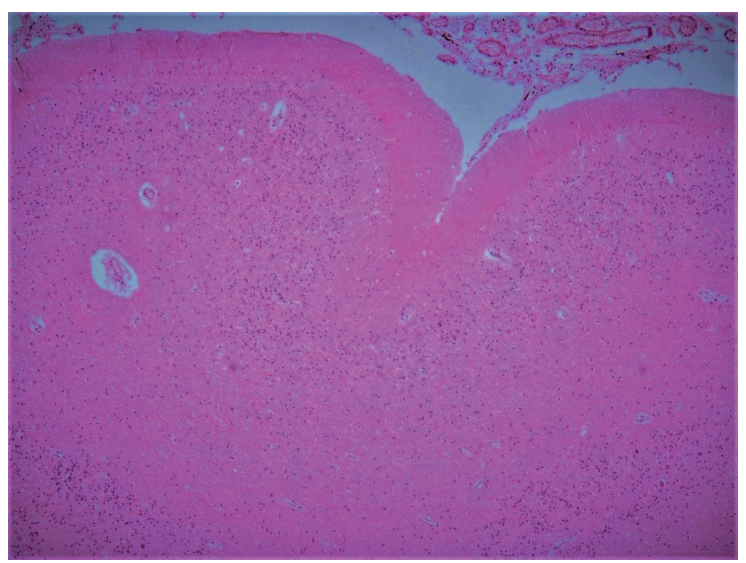

Fig. 1. Cerebellar parenchymal sections showing distinct distortion of molecular and granule cell layers as well as reduced number of Purkinje cells (hematoxlyn-eosin staining). 


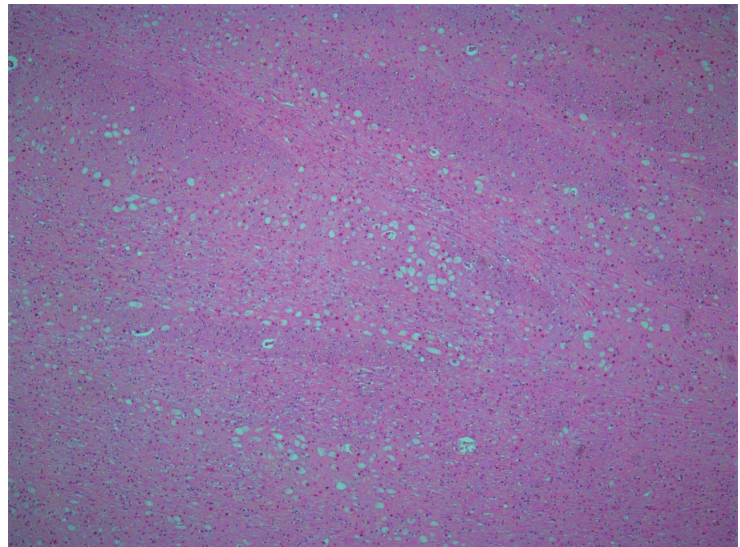

Fig. 2. Groups of large cells which have foamy eosinophylic cytoplasm on brain stem sections (hematoxlyn-eosin staining).

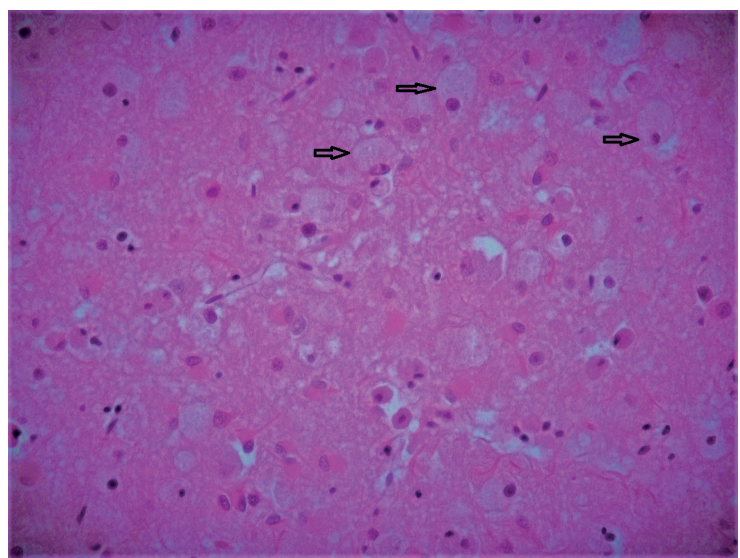

Fig. 3. The cerebral parenchyma was covered by a group of large cells which have foamy eosinophilic cytoplasm (shown with arrows) as well as gemistocytic astrocytes, macrophages and reduced number of normal appearing glial cells (hematoxlyn-eosin staining).

The patient was the first born child of a large family, any other members of which did not show any similar case, according to the family history stated. His mother denied any problems during pregnancy and delivery. His past medical history revealed that the diagnosis of type 1 GM1 gangliosidosis had been established upon his admission for psychomotor retardation, and he had been hospitalized several times for lung infections during the follow-up. Two months before his death, a nasogastric feeding tube had been implemented due to dysphagia.

The autopsy was performed in our institute. External physical examination revealed sunken eyes. Cerebrum and cerebellum weighted 981 g. Serial sections of cerebral and cerebellar tissue samples demonstrated a prominent cortical thinning with a thickness of 0.4 $\mathrm{cm}$, accompanied by ventricular dilatation. Gross examination of the abdomen showed an enlarged liver of $480 \mathrm{~g}$, on the cut surface of which was present frequent spotty yellow areas. Neither any gross pathology of the other organs nor any postmortem toxic substance was detected.

The tissue samples from major organs were obtained by a pathologist. The samples were processed following the standard procedures, H\&E stained, and thereafter evaluated under a light microscope. The cerebral parenchyma was found to host, besides the neural cells, numerous solitary or group-forming cells bearing large, foamy and eosinophilic cytoplasm, as well as many gemistocytic astrocytes with a reduced number or normally appearing glia (Fig. 3). Those large, foamy and eosinophilic cells were also present in the brain stem slides. Cerebellar sections revealed normal histomorphology. Evidence of bronchitis and bronchopneumonia were present in the pulmonary tissue samples. No pathological findings of other organs were detected.

As well as informed consent of families, ethical committee consent was received before the preparation of this article.

\section{Discussion}

Generalized gangliosidosis or GM1 gangliosidosis as more commonly referred to, is associated with the lysosomal accumulation of the monosialoganglioside, GM1. This disease is due to deficiency of the enzyme $ß$-galactosidase, which is inherited in an autosomal recessive fashion. Our first presented case was a child of consanguineous marriage, the mother being 18 years old and having a first cousin who had also suffered GM1 gangliosidosis. However, our second case was the first born child of a non-consanguineous marriage, having no relatives with a similar metabolic disease.

Patients with the infantile form of the disease may show dysmorphic facial features including 
coarse facies, frontal bossing, flat and large nasal bridge, low-set ears, prominent maxilla, large mouth, as well as other findings such as hypotonia, hepatosplenomegaly, bone deformities and macular cherry-red spots 1-4. Along with macrocephaly, these dysmorphic facial features were found to be present in our first case, and both cases had enlarged liver and spleen. However, neither of our cases manifested skeletal deformities or macular cherry-red spots.

Although widespread Mongolian spots are reported to be frequently present on the trunk, back and extremities of the patients with mucopolysaccharidosis and GM1 gangliosidosis, neither of our patients showed these lesions. ${ }^{5,6}$

Three distinct forms of the disease have been described depending on the severity and extent of clinical features, the enzyme levels, and the disease onset. Both cases that are presented here were consisted with the infantile type, with an early childhood onset and abrupt clinical features including rapid neurological deterioration. Being the most severe form, infantile GM1 gangliosidosis possesses a poor prognosis and leads to death during early childhood. ${ }^{7}$ Accordingly, our cases died at the ages of two and three. The juvenile and adult forms, on the contrary, usually appear at later stages of life, and demonstrate a milder clinical course with higher $ß$-galactosidase enzyme levels when compared to the infantile form. ${ }^{1,8,9}$

Initially, cases show hypotonia with brisk deep tendon reflexes; however, within the course of the disease spasticity and flexion contractures become evident. ${ }^{10,11}$ Convulsions may occur after six months., ${ }^{412}$ Both of our patients, according to their past medical records, had sought for medical care for mental and motor developmental problems, underwent thorough evaluation procedures, and had been diagnosed as GM1 gangliosidosis thereafter. Of note, infantile spasticity was present in both patients. Most of the cases die of infections (predominantly pneumonia) or cardiopulmonary dysfunction within the first years of their lives. ${ }^{13}$ Seizures may occur late in the course, and may lead to aspiration of food or gastric contents as well as feeding problems, which eventually causes an even higher risk for lung infections, as was the main cause of death for our both cases. The most commonly encountered cardiovascular system complications are dilated and/or hypertrophic cardiomyopathy and valvulopathy. ${ }^{13}$ Evidence of hypertrophic cardiomyopathy and pulmonary hypertension had been documented on the hospital records for one of the cases reported here.

Lymphocytic vacuolization and foamy macrophages are commonly detected on peripheral blood smears. Additionally, besides the common landmarks of a variety of other metabolic conditions, a histopathological evaluation may also demonstrate macrophages that contain PAS-positive material residing in the liver, spleen, lungs, lymph nodes and bone marrow tissues of GM1 patients. Accumulation of neuronal gangliosides is commonly encountered in glial cells. ${ }^{14,15}$ In our cases, cells with large and foamy cytoplasm were detected in the cerebral, cerebellar and brain stem sections, disrupting the normal histomorphology. Via histochemical staining, PAS-positive macrophages were also detected admixed with neuronal gangliosides (Fig. 4).

Renal tubular cells and placental syncytiotrophoblasts may also show vacuolated appearance and may be a clue for diagnostic approach. Some infantile type cases have a history of non-immune hydrops. ${ }^{16}$ Vacuolated appearance of renal tubular cells were obvious in the renal slides of the second case we evaluated.

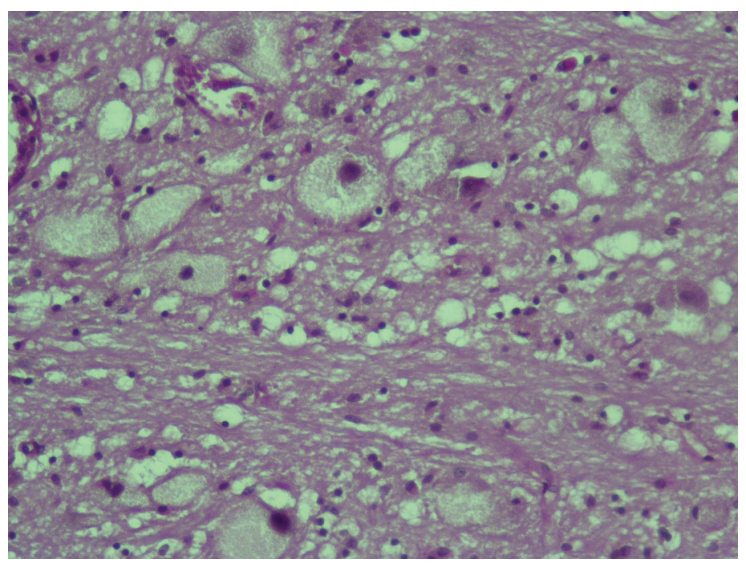

Fig. 4. PAS (periodic acid-Schiff) positive macrophages admixed with neuronal gangliosides. 
Low levels of $ß$-galactosidase enzyme must be documented in leucocytes for a definitive diagnosis. ${ }^{17,18}$ A sampling of amniotic fluid and chorionic villi for enzymatic and/or molecular studies may allow a prenatal detection of the disease. ${ }^{19}$ Unfortunately, no specific treatment is currently available.

As a final word, metabolic and storage disorders should not be overlooked when postmortem examinations of infants are performed. A detailed autopsy and histopathological evaluation may provide some specific evidence that is suggestive of such disorders. We hope, this perspective will aid to define the cause of death in cases without any previous diagnosis.

\section{REFERENCES}

1. Suzuki Y, Oshima A, Nanba E. B-galactosidase deficiency (B-galactosidosis), GM1 gangliosidosis and Morquio B disease. In: Scriver CR, Sly WS, Valle $\mathrm{D}$, et al (eds).The Metabolic and Molecular Bases of Inherited Disease (8th ed). New York: McGraw-Hill Professional, 2001: 3775-3810.

2. Brunetti-Pierri N, Scaglia F. GM1 gangliosidosis: review of clinical, molecular, and therapeutic aspects. Mol Genet Metab 2008; 94: 391-396.

3. Dweikat I, Libdeh BA, Murrar H, et al. GM1 gangliosidosis associated with neonatal-onset of diffuse ecchymoses and mongolian spots. Indian J Dermatol 2011; 56: 98-100.

4. Suzuki K. Neuropathology of late onset gangliosidoses. A review. Dev Neurosci 1991; 13: 205-210.

5. Hanson M, Lupski JR, Hicks J, Metry D. Association of dermal melanocytosis with lysosomal storage disease: clinical features and hypotheses regarding pathogenesis. Arch Dermatol 2003; 139: 916-920.

6. Snow TM. Mongolian spots in the newborn: do they mean anything? Neonatal Netw 2005; 24: 31-33.

7. Pastores GM, Kolodny EH. Lysosomal storage disease. In: Swaiman KF, Ashwall S, Ferriero DM (eds). Pediatric Neurology Principles and Practice (4th ed). Philadelphia: Mosby Elsevier, 2006: 659-714.

8. Roze E, Paschke E, Lopez N, et al. Dystonia and parkinsonism in GM1 type 3 gangliosidosis. Mov Disord 2005; 20: 1366-1369.
9. Celtikçi B, Aydin Hİ, Sivri S, Sönmez M, Topçu $\mathrm{M}$, Ozkara HA. Four novel mutations in the ß-galactosidase gene identified in infantile type of GM1 gangliosidosis. Clin Biochem 2012; 16: 571574.

10. Muthane U, Chickabasaviah Y, Kaneski C, et al Clinical features of adult GM1 gangliosidosis: report of three Indian patients and review of 40 cases. Mov Disord 2004; 19: 1334-1341.

11. Ohto U, Usui K, Ochi T, Yuki K, Satow Y, Shimizu T. Crystal structure of human ß-galactosidase: structural basis of GM1 gangliosidosis and Morquio B diseases. J Biol Chem 2012; 287: 1801-1812.

12. Lenicker HM, Vassallo Agius P, Young EP, Attard Montalto SP. Infantile generalized GM1 gangliosidosis: high incidence in the Maltese Islands. J Inherit Metab Dis 1997; 20: 723-724.

13. Morrone A, Bardelli T, Donati MA, et al. Betagalactosidase gene mutations affecting the lysosomal enzyme and the elastin-binding protein in GM1gangliosidosis patients with cardiac involvement. Hum Mutat 2000; 15: 354-366.

14. Shield JP, Stone J, Steward CG. Bone marrow transplantation correcting beta-galactosidase activity does not influence neurological outcome in juvenile GM1-gangliosidosis. J Inherit Metab Dis 2005; 28: 797-798.

15. Wynn RF, Wraith JE, Mercer J, et al. Improved metabolic correction in patients with lysosomal storage disease treated with hematopoietic stem cell transplant compared with enzyme replacement therapy. J Pediatr 2009; 154: 609-611.

16. Ferreira CR, Gahl WA. Lysosomal stroge diseases. Transl Sci Rare Dis 2017: 2: 1-71.

17. Suzuki Y, Sakuraba H, Oshima A, et al. Clinical and molecular heterogeneity in hereditary betagalactosidase deficiency. Dev Neurosci 1991; 13: 299303.

18. Chamoles NA, Blanco MB, Iorcansky S, Gaggioli D, Spécola N, Casentini C. Retrospective diagnosis of GM1 gangliosidosis by use of a newborn-screening card. Clin Chem 2001; 47: 2068.

19. Severini MH, Silva CD, Sopelsa A, CoelhoJC, Giugliani R. High frequency of type 1 GM1 gangliosidosis in southern Brazil. Clin Genet 1999; 56: 168-169. 